\title{
Simple Scaling Of Multi-Stream Jet Plumes For Aeroacoustic Modeling
}

\author{
James Bridges ${ }^{\dagger}$ \\ NASA Glenn Research Center, Cleveland, $\mathrm{OH} 44070$
}

30 November 2015

\begin{abstract}
When creating simplified, semi-empirical models for the noise of simple single-stream jets near surfaces it has proven useful to be able to generalize the geometry of the jet plume. Having a model that collapses the mean and turbulent velocity fields for a range of flows allows the problem to become one of relating the normalized jet field and the surface. However, most jet flows of practical interest involve jets of two or more coannular flows for which standard models for the plume geometry do not exist. The present paper describes one attempt to relate the mean and turbulent velocity fields of multi-stream jets to that of an equivalent singlestream jet. The normalization of single-stream jets is briefly reviewed, from the functional form of the flow model to the results of the modeling. Next, PIV data from a number of multistream jets is analyzed in a similar fashion. The results of several single-stream approximations of the multi-stream jet plume are demonstrated, with a 'best' approximation determined and the shortcomings of the model highlighted.
\end{abstract}

\section{Nomenclature}

$\begin{array}{ll}A & \text { nozzle area } \\ B P R & \text { mass bypass ratio, all fan flows/core flow } \\ c & \text { speed of sound } \\ D & \text { nozzle diameter } \\ D e & \text { area-equivalent nozzle diameter } \\ L & \text { length from nozzle exit to plug tip } \\ M & \text { Mach number } \\ M a & \text { acoustic Mach number }\left(M a=U_{j} / c_{\infty}\right) \\ N P R & \text { nozzle total pressure ratio } \\ N T R & \text { nozzle total temperature ratio } \\ S S S & \text { Simple Single Stream model } \\ T K E & \text { turbulent kinetic energy } \\ T & \text { temperature } \\ T s R & \text { static temperature ratio, } T_{j} / T_{\infty} \\ U & \text { jet velocity } \\ x & \text { axial coordinate } \\ X & \text { normalized axial coordinate, } x / D \\ \alpha, \beta & \text { model parameters } \\ \phi & \text { mean velocity ratio } \\ \square \square \square \square \square \square \square \square \square \square \\ 1,2,3 & \text { core, bypass, tertiary stream } \\ i, o & \text { inner, outer } \\ j & \text { jet exit } \\ \infty & \text { ambient } \\ m i x & \text { fully mixed } \\ m a x & \text { maximum }\end{array}$

\footnotetext{
$\dagger$ Acoustics Branch, MS 54-3; AIAA Associate Fellow
} 
M obtained by model

$x \quad$ obtained from experiment

\section{Introduction}

In models of noise from simple single-stream jets the exit jet diameter, velocity, and temperature have been found to be adequate to capture the main scaling laws of jet noise. As measured by phased arrays, the distribution of acoustic sources in an isolated jet collapse for a variety of jets when the frequency of the noise is normalized as Strouhal number based on jet diameter and the axial distribution normalized by jet potential core ${ }^{1}$. This characterization is useful when constructing models of jet-surface interaction. Brown ${ }^{2}$ has developed models for how jets interact with surfaces to produce and modify the mixing noise. In these models for simple, single-stream jets, the jet plume was primarily characterized by the length of the jet potential core, which was itself related to jet flow conditions ${ }^{3}$.

Studies such as that of $\mathrm{Witze}^{4}$ and $\mathrm{Lau}^{5}$ have established useful correlations of potential core length to jet flow conditions. However, most jets of interest are comprised of at least two coaxial streams, making the task of modeling the jet, and therefore jet-surface interaction, much more difficult. For starters, it is not clear what constitutes a potential core in a multi-stream jet, and therefore jet geometry and noise source distributions are not as straightforward to predict as in a single-stream jet. Furthermore, when the jet is issuing from a separate flow nozzle with a plug even the origin of the jet is somewhat ambiguous - should the origin be considered the location of the first nozzle lip or the location where the jet diameter is minimal near the end of the plug?

More complicated analyses have been applied to the analysis of the acoustic impact of having a surface near a multi-stream jet. For example, Papamoschou and Rostamimonjezi ${ }^{6}$ used a near-field wave packet model to represent the acoustic source, predicting the change to the far field as the surface geometry is changed and even as enhanced mixing devices on the nozzle lip change the jet geometry. Such approaches have yet to be made simple enough for system-level studies. For a faster method of prediction it would be useful to produce a simple one-dimensional model of the multi-stream jet's geometry that could guide acoustic modeling of jet-surface interactions. Such a model at its simplest might relate the flow conditions of the multi-stream jet plume to that of an equivalent single-stream jet.

Assuming that the key quantities in aeroacoustic modeling are exit acoustic Mach number $\left(M a=V_{j} / c_{\infty}\right)$ and turbulent kinetic energy (TKE), the simplest way of relating the noise of multi-stream jets to that of simple single stream jets is through mapping of $M a$ and $T K E$ from one to the other. We want to rescale the jet noise models by finding an equivalent distribution of $M a$ and $T K E$ for the multi-stream jets. This entails finding an equivalent diameter, an equivalent $M a$, an equivalent jet lengthscale (potential core length), and a peak $T K E$ for the multi-stream jets. In the case of separate flow nozzles and nozzles with external plugs in general there is the additional problem of finding an equivalent nozzle origin to relate to the simple single opening of the single-stream jet.

Recently, Henderson and Wernet acquired particle image velocimetry (PIV) data for a range of two- and threestream nozzle flows ${ }^{7}$. The primary variations in the experiment were nozzle area ratios and fan stream pressure ratios. A substantial number of axisymmetric cases were acquired, most with forward flight. The matrix of flow conditions and nozzle geometries were designed to match three-stream variable-cycle engines being considered for future applications. The wide range of flows measured also makes this a good database to use in modeling multi-stream jet plumes.

In the current paper this rich database is analyzed, attempting to find equivalence between multi-stream jets from complicated nozzles and single-stream jets from simple nozzles. The presentation starts with a review of a model characterizing the flow fields of simple, single-stream jets. This section uses a more robust method of fitting a model for the jet centerline profile than was previously published ${ }^{3}$ for the single-stream NASA PIV database ('Consensus Dataset'). The fitted parameters result is a model for the centerline velocity of a jet in terms of jet diameter, exit velocity, and temperature. The next section documents the particular multi-stream nozzles and flow conditions that are analyzed in the remainder of the paper, extracted from the larger study of Henderson and Wernet ${ }^{7}$. The analysis which follows consists of (i) applying the fitting method to the multi-stream jet data to determine potential core length and exponential decay rate, (ii) postulating various equivalences between jet diameter, velocity, and temperature that will make the simple, single-stream model best match the complex, multi-stream experimental data, and (iii) comparing the model and experimental parameters to measure success of the postulated equivalences. Direct comparisons of the centerline mean velocity profiles, data versus simple model, are provided for a more physical assessment of the discrepancies. The last section uses these parametric values of potential core length and decay rate to attempt a collapse of the turbulent velocity of the jets, thought to be a key aspect of jet noise and jet surface interaction noise. 


\section{Plume modeling for single-stream, simple jets}

The method for creating the model for axial decay of centerline velocity from experimental data is relatively straightforward. From plots of the streamwise PIV data the potential core length and centerline decay rate, along with the peak TKE are determined. The mean velocity decay parameters are determined using the same method used on single-stream jets to develop a Witze-like model. The model for jet velocity $U$ on its centerline takes the form

$$
\phi=1-\exp \left\{\frac{\alpha}{1-\frac{x}{\beta}}\right\}
$$

where $\phi$ is the normalized mean axial velocity,

$$
\phi=\left(U-U_{\infty}\right) /\left(U_{j}-U_{\infty}\right) .
$$

$X$ is the non-dimensional axial distance $x / D, D$ is the jet exit diameter, $U_{j}$ and $U_{\infty}$ are the jet exit velocity and ambient velocity, respectively, and parameters $\alpha$ and $\beta$ are determined by the flow conditions. In this functional form $\beta$ determines potential core length while $\alpha$ specifies the exponential decay rate beyond the potential core. Values for the parameters $\alpha$ and $\beta \square$ we $\square \square \square \square \square \square \square \square \square \square \square \square \square$ by fitting the centerline velocity profile of Eq [1] to the experimental data in a manner outlined in Section A below. These parameters are then modeled as functions of the flow variables to find a universal model for jet centerline decay. This replicates the work of Witze ${ }^{4}$, with more modern and accurate data, and without assuming that all jets have the same decay rate. In Witze's paper, the decay rate Xc (equivalent to $\alpha$ in the present work) is assumed to be a universal constant, equal to 1.4. The potential core length is called $\mathrm{x}_{\text {core }}$ in Witze, and is equivalent to $\beta$ here.

In previous work with the NASA PIV Consensus Database ${ }^{3,8}$, the centerline velocity profiles of a wide range of single-stream jets were collected, as summarized by the plot on the left-hand side of Figure 1 . The range of jet conditions that are included in the dataset, $0.5<M a<1.33,0.84<T s R<2.37$. In that paper, the data were normalized by potential core length, following Witze, and shown to have a reasonable collapse as shown on the right-hand plot of Figure 1. Since that publication, application of formal fitting methods to include variable decay rate $\alpha$, as described below, resulted in a much-improved collapse of data (Figure 2). Not only were the mean velocities effectively normalized, but the TKE profiles along the centerline and the lipline were also brought to a neat collapse (Figure 3).

Formulas were developed for these parameters as functions of jet Mach number $M_{j}$ and static temperature ratio $\operatorname{Ts} R\left(=T_{j} / T_{\infty}\right)$ :

$$
\begin{aligned}
& \alpha=\frac{-1.848\left(1-0.25 M_{j}\right)}{T s R^{0.4}} \\
& \beta=\frac{3.195\left(1+0.796 M_{j}\right)}{T s R^{0.11}}
\end{aligned}
$$

The formulas for $\alpha$ and $\beta$ given in Eqs [3,4] will be referred to as the Simple Single-Stream model, or SSS model.

The current paper starts with an overview of test facilities, test articles, and instrumentation used to collect the jet flow data. Velocity data extracted from measurements of a range of multi-stream jets is presented and the analysis performed on their flow fields. Various ways of approximating the parameters of the multi-stream jet as a single jet are introduced and the single-stream model applied using these approximations. 

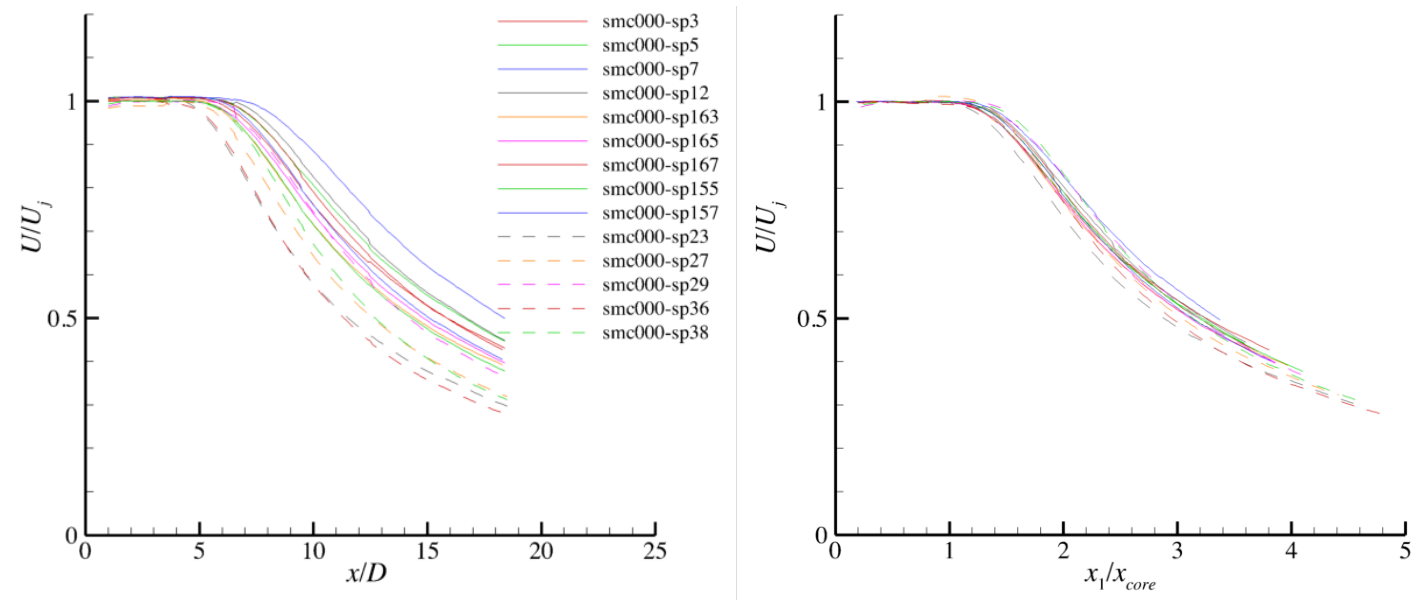

Figure 1 Centerline profile of mean velocity, (left) as measured and (right) rescaled by Witze's model for potential core length $x_{\text {core. }}$ Jet flows covering $0.5<M a<1.33,0.84<T s R<2.37$, given in Table 1 .

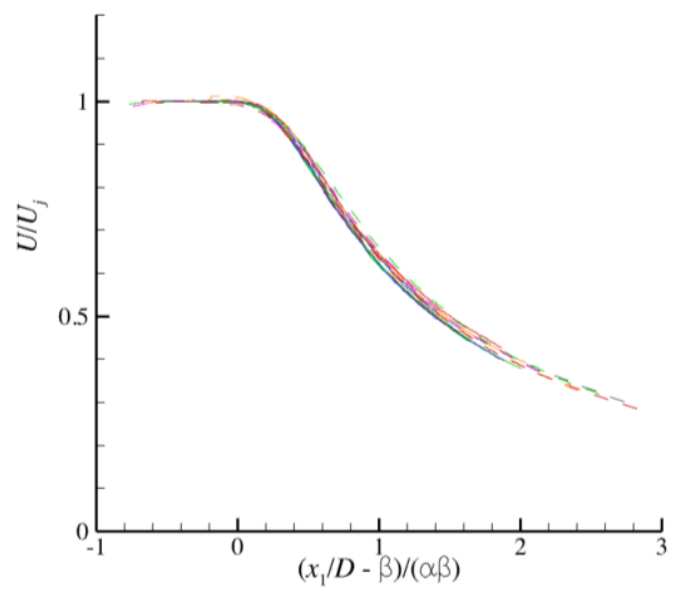

Figure 2 Centerline profile of mean velocity rescaled by SSS model parameters $\alpha$, $\beta$. Jet flows covering $0.5<$ $M a<1.33,0.84<T s R<2.37$, given in Table 1 .
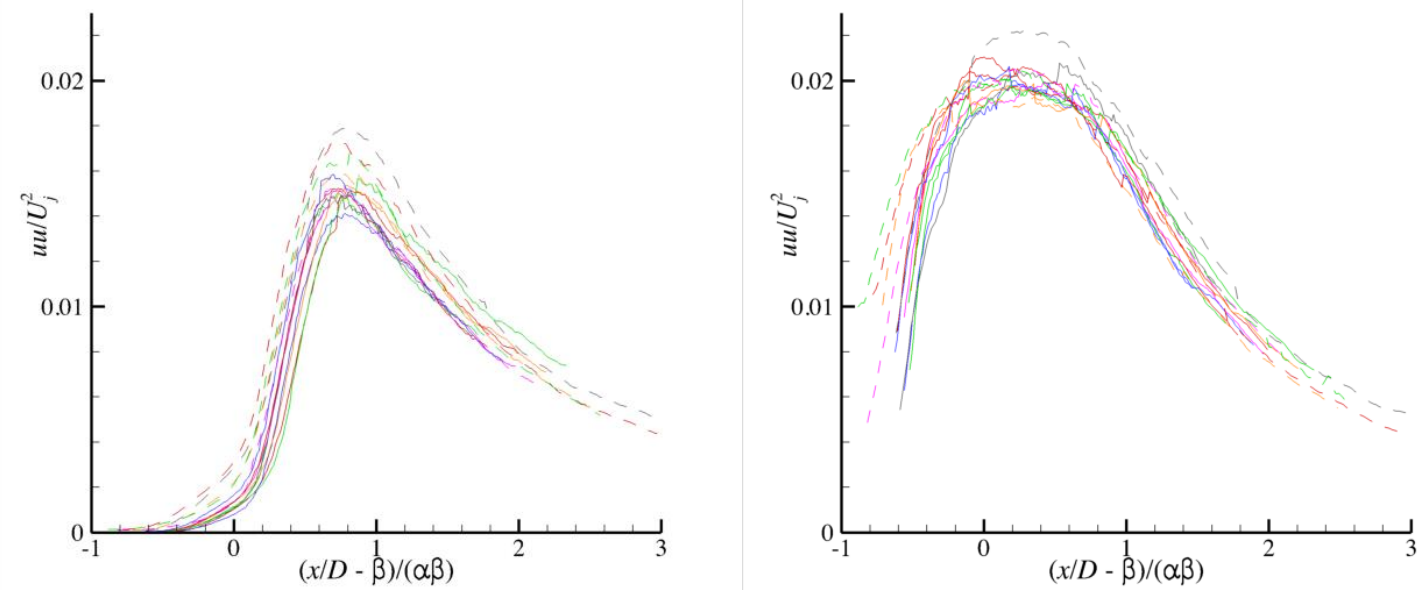

Figure 3. Centerline (left) and lipline (right) profiles of turbulent axial velocity, rescaled by SSS model parameters $\alpha$, $\beta$. Jet flows covering $0.5<M a<1.33,0.84<T s R<2.37$, given in Table 1 . 
Table 1 Jet flows used in SSS model, and parameters of nondimensionalization of axial coordinate.

\begin{tabular}{ccccccc}
\hline Nozzle Type & Setpoint & $\boldsymbol{M}$ & $\boldsymbol{M a}$ & $\boldsymbol{T}_{\boldsymbol{j}} / \boldsymbol{T}_{\infty}$ & $\boldsymbol{\alpha}$ & $\boldsymbol{\beta}$ \\
\hline Convergent & 3 & 0.51 & 0.5 & 0.95 & 0.777 & 4.47 \\
Convergent & 5 & 0.74 & 0.7 & 0.89 & 0.656 & 5.21 \\
Convergent & 7 & 0.98 & 0.9 & 0.84 & 0.595 & 6.21 \\
Convergent & 12 & 0.90 & 0.9 & 1 & 0.590 & 5.64 \\
Convergent & 163 & 0.48 & 0.5 & 1.1 & 0.661 & 4.52 \\
Convergent & 165 & 0.67 & 0.7 & 1.1 & 0.618 & 4.94 \\
Convergent & 167 & 0.86 & 0.9 & 1.1 & 0.660 & 5.04 \\
Convergent & 155 & 0.64 & 0.5 & 1.2 & 0.571 & 4.95 \\
Convergent & 157 & 0.82 & 0.9 & 1.2 & 0.593 & 5.14 \\
Convergent & 23 & 0.38 & 0.5 & 1.76 & 0.548 & 4.09 \\
Convergent & 27 & 0.68 & 0.9 & 1.76 & 0.495 & 4.86 \\
Convergent & 29 & 1.01 & 1.33 & 1.76 & 0.441 & 5.83 \\
Convergent & 36 & 0.60 & 0.9 & 2.27 & 0.448 & 4.57 \\
Convergent & 38 & 0.89 & 1.33 & 2.27 & 0.399 & 5.54 \\
\hline
\end{tabular}

\section{Data}

Particle image velocimetry of the multi-stream jets was acquired in the Nozzle Acoustic Test Rig (NATR) of the Aero-Acoustic Propulsion Laboratory of NASA's Glenn Research Center, and is being reported by Henderson and Wernet ${ }^{7}$. Extensive datasets were acquired, both in streamwise and cross-stream planes, for a wide range of flows on separate flow, plugged, nozzles with three coannular streams. Only a subset of the streamwise data was used in this study. Please see the cited reference for details of the facility, test hardware, and instrumentation used in the acquisition of the data analyzed herein.

The flow conditions of the three streams were completely characterized by multi-element rakes in the jet rig and flow venturii in the supply lines, providing direct measurement of the nozzle plenum total pressure and temperature. Ambient conditions were also continuously recorded. From these measurements the ideally expanded velocity, static temperature, mass flowrate, acoustic Mach number, and temperature ratio could be directly computed, and the measured nozzle areas confirmed. These flow values were computed for each stream (denoted with the subscripts 1 , 2, 3 for core, bypass, or tertiary) and for mass-weighted equivalents (denoted with the subscript mixed). The subscript $e$ is used on area or diameter to mean total or equivalent area and diameter.

\section{A. Nozzle geometries}

In the centerline decay model of Eq [1], a jet velocity, a jet diameter, and a nozzle origin are required as input. If the SSS model parameters $\alpha$ and $\beta$ are to be used, a jet temperature and ambient temperature must also be specified. For multi-stream jets two candidate jet velocities come to mind: the maximum velocity (typically the core stream) and the mass-weighted, fully mixed velocity. For an externally mixed nozzle, two jet diameters are obvious, the external nozzle diameter (which sets the initial shear layer area) and a jet diameter that has equal exit area (which better mimics the downstream plume). Another diameter previously employed ${ }^{9}$ in multi-stream jets is the mass flow equivalent diameter, defined as $D e_{m}=D e_{1} \sqrt{\left(\dot{m}_{1}+\dot{m}_{2}\right) / \dot{m}_{1}}$. For a nozzle origin, the initial nozzle exit could be chosen, presumably corresponding to the choice of outer nozzle diameter as jet diameter. Alternatively, a fictitious origin corresponding to the necked down plume near the end of the plug could be chosen. This would be sensible if an equivalent area diameter is chosen.

Three nozzle configurations, listed in Table 2, were used during the PIV testing. Quantities used in the modeling effort, such as area-equivalent diameters and axial locations of nozzle lips relative to the plug tip, are given in Table 3. Visual representations of the nozzles are given in Figure 4. Two of the configurations, C3T1 and C3T3 are visually indistinguishable, being different by only a slight difference in the third stream exit diameter. 
Table 2 Nozzle dimensions, diameters and areas $(\mathbf{m m})$.

\begin{tabular}{cccccccccc}
\hline Config & $\boldsymbol{D}_{\boldsymbol{1} \boldsymbol{i}}$ & $\boldsymbol{D}_{\boldsymbol{1 , \boldsymbol { o }}}$ & $\boldsymbol{A}_{\boldsymbol{1}}$ & $\boldsymbol{D}_{2, \boldsymbol{i}}$ & $\boldsymbol{D}_{2, \boldsymbol{o}}$ & $\boldsymbol{A}_{\boldsymbol{2}}$ & $\boldsymbol{D}_{3, \boldsymbol{i}}$ & $\boldsymbol{D}_{3, \boldsymbol{o}}$ & $\boldsymbol{A}_{\boldsymbol{3}}$ \\
\hline C1T1 & 93.3 & 131.7 & 6793 & 187.2 & 238.1 & 16993 & 279.4 & 294.0 & 6566 \\
C3T1 & 93.3 & 131.7 & 6793 & 219.2 & 238.1 & 6778 & 279.4 & 294.0 & 6566 \\
C3T3 & 93.3 & 131.7 & 6793 & 219.2 & 238.1 & 6778 & 279.4 & 287.6 & 3642 \\
\hline
\end{tabular}

Table 3 Nozzle dimensions, equivalent areas and axial locations from plug tip (mm).

\begin{tabular}{ccccccccc}
\hline Config & $\boldsymbol{A}_{\boldsymbol{2}} / \boldsymbol{A}_{\boldsymbol{1}}$ & $\boldsymbol{A}_{\boldsymbol{3}} / \boldsymbol{A}_{\boldsymbol{1}}$ & $\boldsymbol{D e}_{\boldsymbol{1}}$ & $\boldsymbol{D e}_{\boldsymbol{2}}$ & $\boldsymbol{D} \boldsymbol{e}_{\boldsymbol{3}}$ & $\boldsymbol{L}_{\boldsymbol{1}}$ & $\boldsymbol{L}_{\boldsymbol{2}}$ & $\boldsymbol{L}_{\boldsymbol{3}}$ \\
\hline C1T1 & 2.5 & 1 & 93.0 & 174.0 & 196.6 & 150 & 260 & 325 \\
C3T1 & 1 & 1 & 93.0 & 131.4 & 160.1 & 150 & 320 & 385 \\
C3T3 & 1 & 0.6 & 93.0 & 131.4 & 148.0 & 150 & 320 & 385 \\
\hline
\end{tabular}
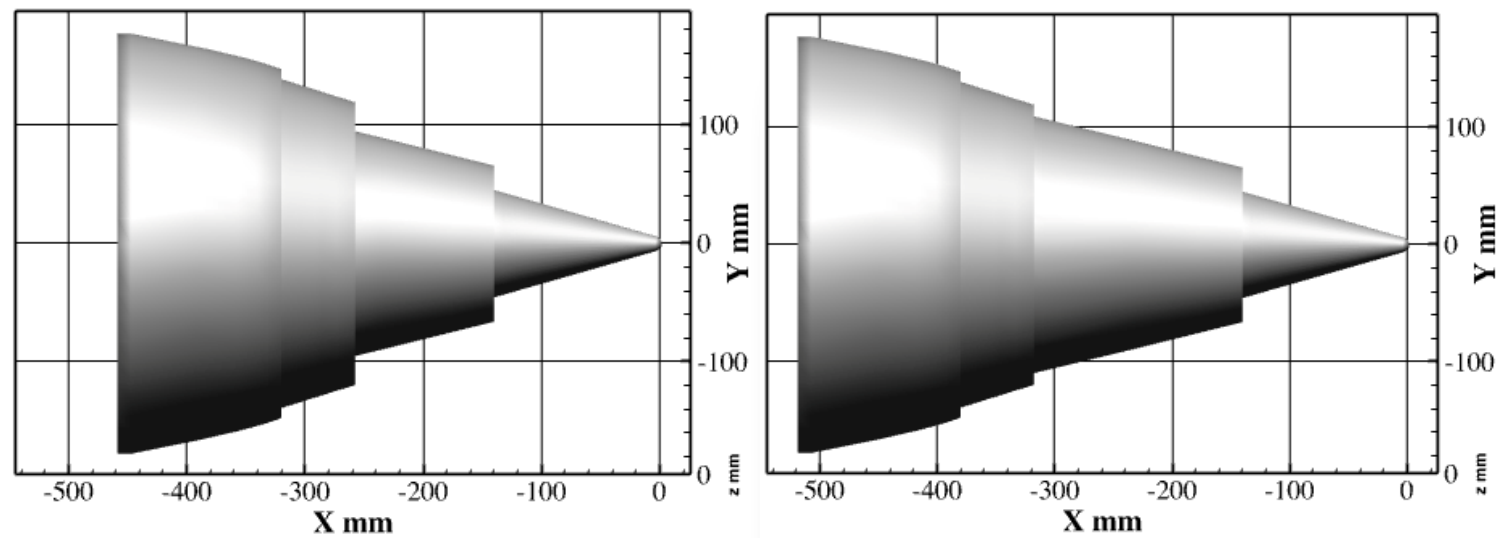

Figure 4. Three-stream nozzles used. C1T1 on left, C3T1 (C3T3) on right.

\section{B. Flow conditions}

The flow condition matrix was based on having a hot, slightly subsonic, core flow $\left(N P R_{1}=1.8, N T R_{1}=3\right)$, a pressurematched second stream $\left(N P R_{2}=1.8, N T R_{2}=1.25\right)$, and a range of third stream flow conditions $\left(N P R_{3}=1.0,1.4,1.8,2.1\right.$; $N T R_{3}=1.25$ ). These were run on a majority of the nozzles (fewer on the C3T3 nozzle). There were a few cases where the second stream was varied $\left(N P R_{2}=1.6\right)$, and two cases where the core flow was run alone, hot and unheated. Most flows had ambient flight flow at $M_{\infty}=0.3$, but some were static. The test cases available are listed in Table 4 by setpoint and nozzle name along with their defining pressure ratio and temperature ratio values. Derived quantities are listed in Table 5. 
Table 4 Axisymmetric test cases available for analysis.

\begin{tabular}{cccccccc}
\hline nozID & setpoint & $\boldsymbol{N P R}_{\boldsymbol{1}}$ & $\boldsymbol{N P R}_{\mathbf{2}}$ & $\boldsymbol{N P R}_{\mathbf{3}}$ & $\boldsymbol{N T R}_{\boldsymbol{1}}$ & $\boldsymbol{N T R}_{\mathbf{2}}=\boldsymbol{N T R}_{\mathbf{3}}$ & $\boldsymbol{M}_{\infty}$ \\
\hline C1T1 & 88033 & 1.8 & 1.8 & 1 & 3 & 1.25 & 0.3 \\
C1T1 & 88433 & 1.8 & 1.8 & 1.4 & 3 & 1.25 & 0.3 \\
C1T1 & 88833 & 1.8 & 1.8 & 1.8 & 3 & 1.25 & 0.3 \\
C1T1 & 88133 & 1.8 & 1.8 & 2.1 & 3 & 1.25 & 0.3 \\
C1T1 & 88430 & 1.8 & 1.8 & 1.4 & 3 & 1.25 & 0 \\
C3T1 & 80010 & 1.8 & 1 & 1 & 1 & 1.25 & 0 \\
C3T1 & 80030 & 1.8 & 1 & 1 & 3 & 1.25 & 0 \\
C3T1 & 86010 & 1.8 & 1.6 & 1 & 1 & 1.25 & 0 \\
C3T1 & 86210 & 1.8 & 1.6 & 1.2 & 1 & 1.25 & 0 \\
C3T1 & 88033 & 1.8 & 1.8 & 1 & 3 & 1.25 & 0.3 \\
C3T1 & 88433 & 1.8 & 1.8 & 1.4 & 3 & 1.25 & 0.3 \\
C3T1 & 88833 & 1.8 & 1.8 & 1.8 & 3 & 1.25 & 0.3 \\
C3T1 & 88133 & 1.8 & 1.8 & 2.1 & 3 & 1.25 & 0.3 \\
C3T1 & 88430 & 1.8 & 1.8 & 1.4 & 3 & 1.25 & 0 \\
C3T3 & 88033 & 1.8 & 1.8 & 1 & 3 & 1.25 & 0.3 \\
C3T3 & 88433 & 1.8 & 1.8 & 1.4 & 3 & 1.25 & 0.3 \\
C3T3 & 88133 & 1.8 & 1.8 & 2.1 & 3 & 1.25 & 0.3 \\
\hline
\end{tabular}

Table 5 Derived flow quantities for test cases.

\begin{tabular}{lcccccccc}
\hline nozID & setpoint & $\begin{array}{c}\boldsymbol{U}_{\boldsymbol{j}} \\
(\mathbf{m} / \mathbf{s})\end{array}$ & $\begin{array}{c}\boldsymbol{U}_{\infty} \\
(\mathbf{m} / \mathbf{s})\end{array}$ & $\begin{array}{c}\boldsymbol{U}_{\boldsymbol{m i x}} \\
(\mathbf{m} / \mathbf{s})\end{array}$ & $\boldsymbol{M a} \boldsymbol{m i x}$ & $\boldsymbol{T s}_{\boldsymbol{s} \boldsymbol{m i x}}$ & $\boldsymbol{B P R}$ & $\boldsymbol{U}_{2} / \boldsymbol{U}_{\mathbf{1}}$ \\
\hline C1T1 & 88033 & 521 & 102 & 378 & 1.11 & 1.37 & 3.86 & 0.02 \\
C1T1 & 88433 & 520 & 103 & 358 & 1.05 & 1.33 & 4.93 & 0.49 \\
C1T1 & 88833 & 521 & 103 & 369 & 1.08 & 1.29 & 5.41 & 0.64 \\
C1T1 & 88133 & 521 & 102 & 381 & 1.12 & 1.27 & 5.65 & 0.71 \\
C1T1 & 88430 & 522 & 16 & 360 & 1.06 & 1.33 & 4.84 & 0.49 \\
C3T1 & 80010 & 315 & 19 & 318 & 0.93 & 0.98 & 0.04 & 0.04 \\
C3T1 & 80030 & 520 & 18 & 405 & 1.19 & 2.06 & 0.47 & 0.02 \\
C3T1 & 86010 & 315 & 17 & 308 & 0.91 & 1.00 & 0.87 & 0.04 \\
C3T1 & 86210 & 315 & 17 & 291 & 0.85 & 1.02 & 1.34 & 0.57 \\
C3T1 & 88033 & 520 & 103 & 415 & 1.22 & 1.66 & 1.38 & 0.01 \\
C3T1 & 88433 & 520 & 104 & 367 & 1.08 & 1.48 & 2.48 & 0.49 \\
C3T1 & 88833 & 520 & 103 & 385 & 1.13 & 1.41 & 2.96 & 0.64 \\
C3T1 & 88133 & 521 & 103 & 402 & 1.18 & 1.37 & 3.28 & 0.72 \\
C3T1 & 88430 & 520 & 17 & 368 & 1.08 & 1.47 & 2.51 & 0.49 \\
C3T3 & 88033 & 520 & 102 & 415 & 1.22 & 1.69 & 1.43 & 0.04 \\
C3T3 & 88433 & 522 & 102 & 391 & 1.15 & 1.59 & 1.91 & 0.49 \\
C3T3 & 88133 & 520 & 102 & 411 & 1.21 & 1.47 & 2.48 & 0.71 \\
\hline
\end{tabular}

\section{Extracting centerline profiles from multi-stream PIV data}

Two features of the flow that complicate analysis are the wake of the plug on the centerline and the slight droop in the peak velocity point downstream. Both features are readily apparent in Figure 5. The simple model for centerline velocity of the jet was derived from cases without these features, coming from a simple single-stream jet. The reason for the plug wake is readily apparent. The reason for the asymmetry is probably more because of the wake of the air supply strut entering from the bottom of the rig a few diameters upstream of the nozzle, which pulled the jet plume downward. This is readily apparent in Figure 5, which shows how having an uneven ambient velocity causes a skew in the cross-stream profiles of mean axial velocity. 

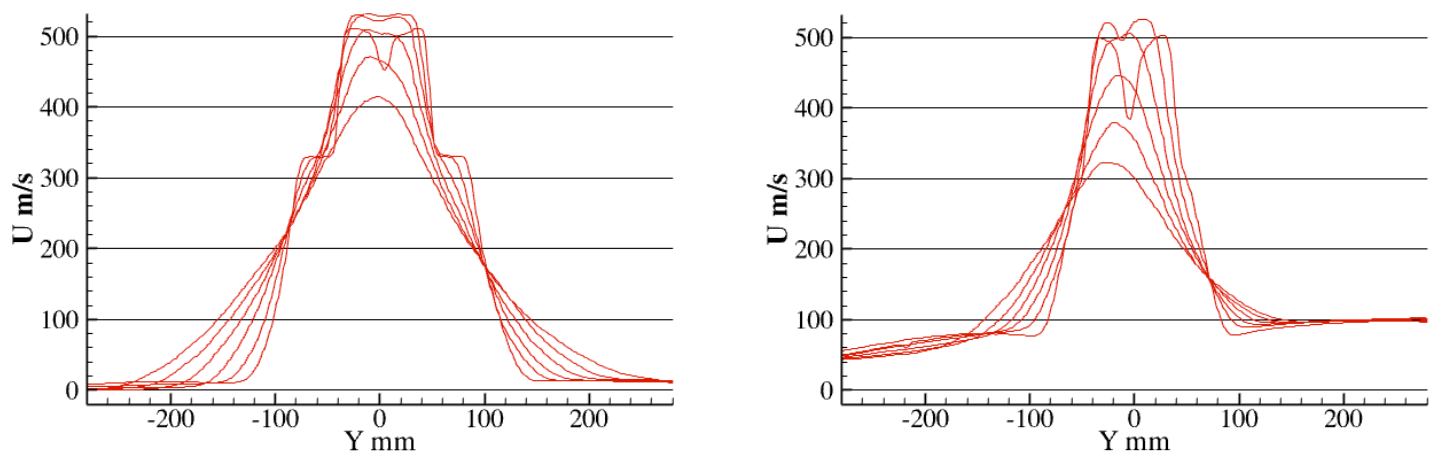

Figure 5 Examples of cross-stream profiles, from plug tip to end of domain. Static case (left) shows no skew in ambient, and flight case (right) shows significant skew.

To obtain data representing the jet centerline, an adjustment was employed. Given that the jet plume seems to deviate slightly from the nozzle centerline, another approach is to extract the data along a pseudo-centerline, defined by a line from the plug center to the radial coordinate containing the maximum velocity at the end of the domain. This line is shown in the Figure 6. Because the pseudo-centerline follows the wake it never reaches the maximum velocity of the core nozzle, but it does maintain a near-constant velocity before making a strong break to a decay that matches the true centerline near the potential core terminus and the peak velocity at the end of the region (by design). Note that by including the plug wake the data will not match the model (Eq [1]) throughout the potential core region! However, the data do clearly delineate the terminus of the potential core. The extracted 'centerline' profiles for all the flows analyzed in this paper are shown in Figure 7. Note the spread of the profiles-it is the intention of the following modeling to create a model that collapses all these profiles.

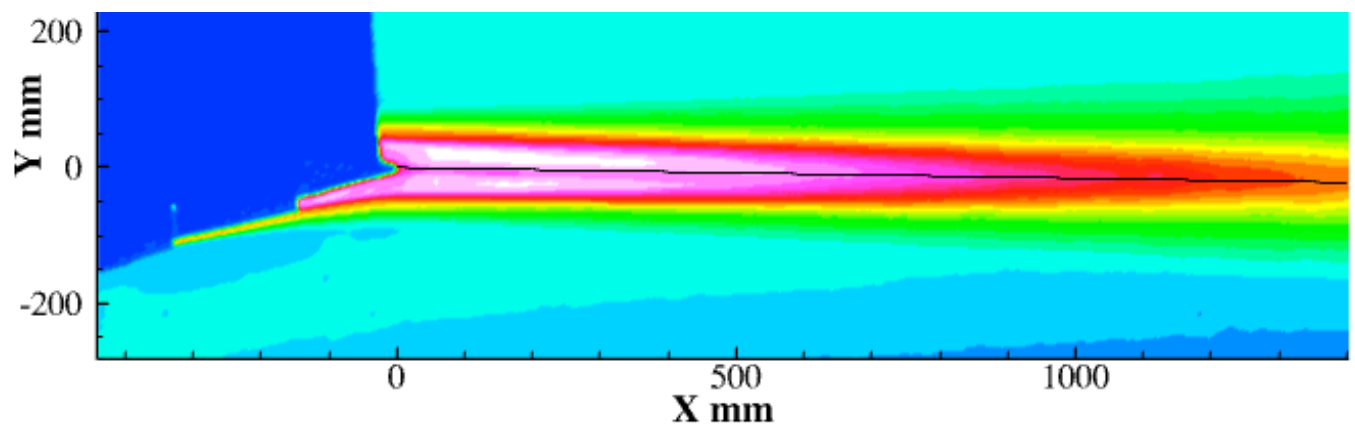

Figure 6. Typical mean axial velocity field from PIV. Line drawn at pseudo-centerline where data was extracted. Axial coordinate origin is the plug tip. 


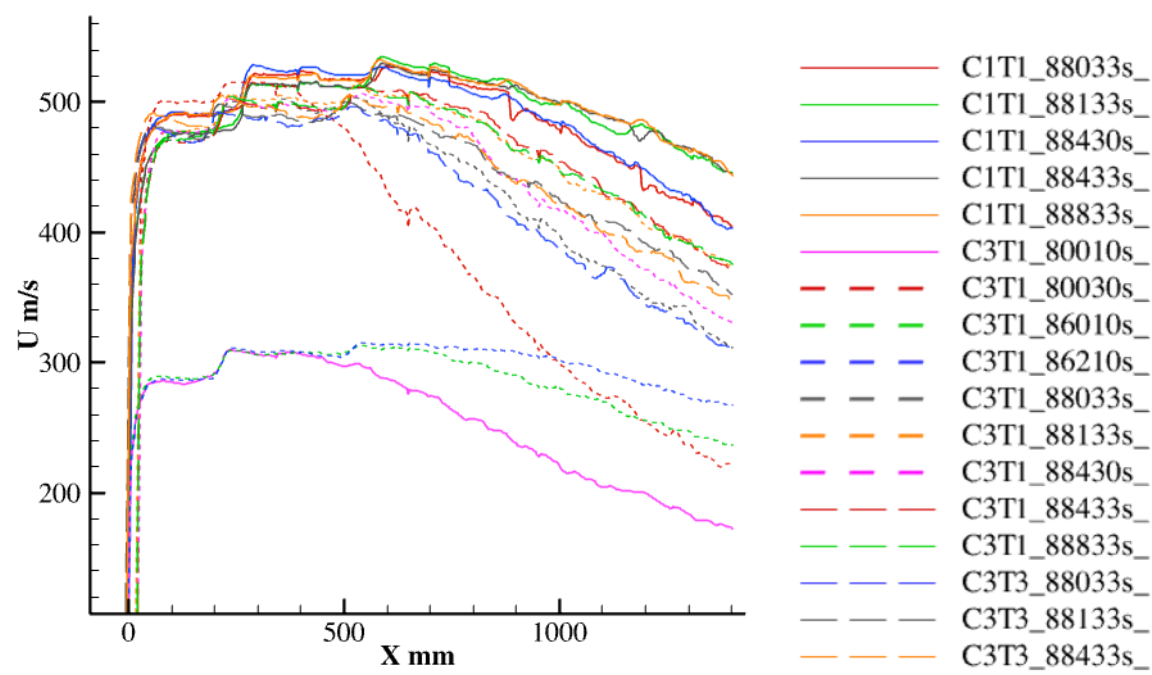

Figure 7 Extracted pseudo-centerline profiles from PIV data in physical coordinates. Axial coordinate origin is the plug tip.

\section{Analysis}

\section{A. Fitting centerline mean velocity data to centerline decay model}

Parameters $\alpha$ and $\beta$ appropriate for a model of multi-stream jets in the form of Eq [1] were determined by finding a linear fit to a transformed version of the data from the decay region as explained next. Equation [1] can be transformed by taking natural log of the equation to the form

$$
\alpha=\left[1-\frac{X}{\beta}\right] \ln (1-\phi)
$$

When [5] is put in the form

$$
X \ln (1-\phi)=\beta \ln (1-\phi)-\alpha \beta
$$

$\alpha \square \square \square \square \square \beta$ can be found by plotting $X \ln (1-\phi)$ vs $\ln (1-\phi)$ and fitting a line to the data. The slope of the fitted line is $\beta$, the intercept is $-\alpha \beta$. By omitting data where $\phi$ is greater than $\sim 0.9$ (the potential core and the round off to the exponential decay region) only the decay region is being fitted. When substantial decay region exists within the field of measurement a robust fit is easily obtained. When the measurements do not extend much beyond the end of the potential core there is potential for bias from the rounding over of the centerline profile. An example of this transformed data and the fit of Eq [6] is given in Figure 8.

Figure 9 is a plot of the centerline mean velocity profiles shown in Figure 7, only normalized by the fitted plume parameters $\alpha$ and $\beta$. The high degree of collapse of the data in Figure 9 shows that the centerline decay downstream of the potential core follows the well-established form of a single stream jet, which was demonstrated in Figure 1. Note that data upstream of $\beta=0$ have been suppressed in the figure, being reflective of the plug wake and not the jet asymptotic scaling. In most jets the wake decayed before the end of the potential core; most curves include a small portion of $\phi$ near 1 just before the roll over to the asymptotic decay. But the fitting does not require that any of the data include the potential core, only the decay portion of the jet centerline and a prescription of the jet's ideally expanded velocity.

While the collapse of the curves is very satisfying, it does not directly lead to a model for multi-stream jet plumes because the values for the parameters $\alpha$ and $\beta$ must be expressed as functions of the jet flow conditions. To create such a model would be difficult with this data set as it does not contain enough variation in the flow conditions for all streams. In fact, the prospect of creating a model for each parameter as a function of at least three jet condition values (velocity, temperature, mass flow fraction) for three streams is daunting (a nine-dimensional variable space!). Instead, the problem was turned around to look for a set of equivalent single-stream jet parameters, constructed from the multi- 
stream nozzle and flow conditions, that could be used for $\alpha$ and $\beta$ which would give a reasonable collapse of the multistream datasets.

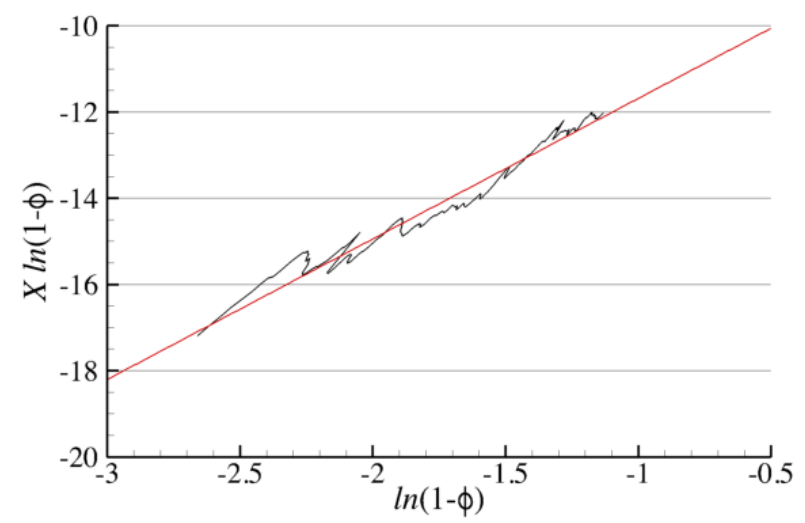

Figure 8 Fitting pseudo-centerline profiles to determine model parameters $\alpha$ and $\beta$.
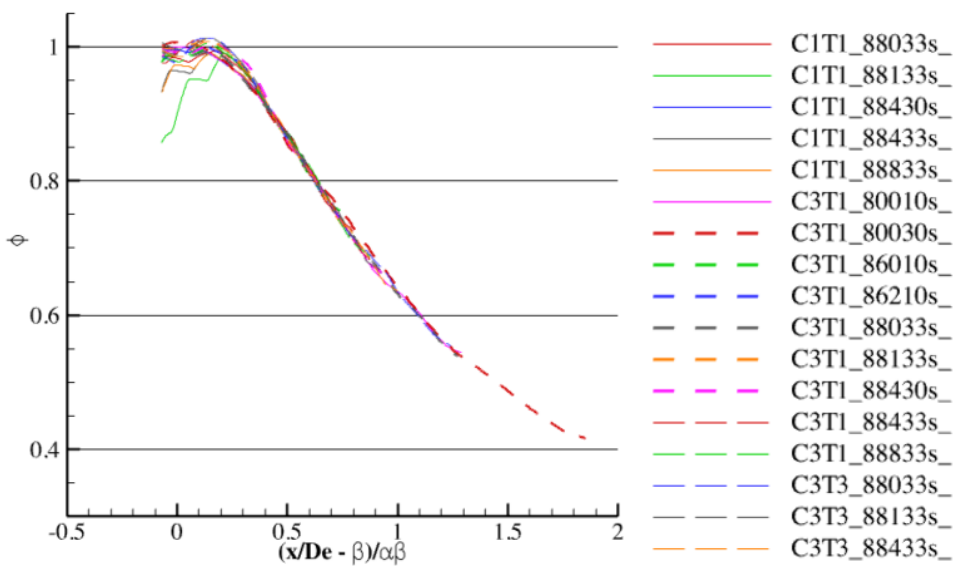

Figure 9 Collapse of mean velocities along centerline when normalized by fitted coefficients $\alpha$ and $\beta$ selecting core nozzle exit and total area-equivalent diameter for scaling of axial coordinate.

\section{B. Choice of equivalent flow conditions relating multi-stream to single-stream jets}

Application of the SSS model to multi-stream jets requires defining nozzle parameters for the origin and scaling of $X$, for $U_{j}$ and $U_{\infty}$ to define $\phi$ (see Eq [2]), and $M a$ and $T s R$ to compute $\alpha$ and $\beta$ (see Eqs [3, 4]). The origin could be chosen as the upstream-most nozzle or it could be chosen to relate to the minimum jet plume diameter that occurs near the tip of the plug. Similarly, the jet diameter could be related to the total nozzle area, a mass-equivalent diameter, or, if one notes that the potential core is that of the inner stream jet embedded in the secondary jet, the equivalent diameter could be the core nozzle diameter. In scaling the velocities there is little choice in $U_{j}$ but $U_{\infty}$ could be chosen to be either the second stream velocity (corresponding to choosing the diameter to be the inner nozzle only) or the ambient velocity (corresponding to choosing the diameter to include all the flows). Finally, an equivalent acoustic Mach number and static temperature ratio must be determined for the calculation of $\alpha$ and $\beta$. This could correspond to the core flow values, a mass-weighted (fully mixed) average, or perhaps a $M a$ defined by the relative velocity of the jet. Table 6 summarizes the possible axial origins $x_{0}$ and jet diameters $D e$ to be used in defining $X=\left(x-x_{0}\right) / D e$ in Eq [6] and $M a$ and $T s R$ in equations [3] and [4]. 
Table 6 Possible choices of axial origin, diameter, and flow scaling.

\begin{tabular}{cl|cl|ll}
\hline & Axial origin, $\boldsymbol{x}_{\boldsymbol{\theta}}$ & \multicolumn{2}{|c|}{ Equivalent diameter, $\boldsymbol{D}_{\boldsymbol{e}}$} & \multicolumn{2}{c}{ Flow conditions, $\boldsymbol{M a}, \boldsymbol{T} \boldsymbol{s}$} \\
\hline$L_{1}$ & Exit of stream 1 & $D e_{1}$ & $A_{l}$ equivalent & $M a_{1}, T s R_{1}$ & Max \\
$L_{\mathrm{t}}$ & Exit of first upstream & $D e_{\text {mix }}$ & Total mixed mass equivalent & $\left(U_{1}-U_{\infty}\right) / \mathrm{c}_{\infty}, T s R_{1}$ & Max-inf \\
& $D e_{\mathrm{t}}$ & Total nozzle area equivalent & $M a_{m i x}, T s R_{m i x}$ & Mixed \\
\hline
\end{tabular}

For this exercise $U_{\infty}$ was taken to be the ambient velocity, either $M_{\infty}=0.0$ or 0.3 . The PIV data were normalized by all combinations of axial origin and jet diameter listed in Table 6 and by the parameters $\alpha$ and $\beta$ computed from the SSS model, Eqs [3,4], using the three choices of flow conditions $M a$ and $T s R$ shown in Table 6. The success of the modeling would be determined by the degree to which these normalizations collapsed the PIV data from all the different nozzles and flows. For brevity, only the more successful combinations are provided here; normalizing by $D e_{1}$ did not work well and no examples will be shown.

Two figures have been prepared to show the results of trying the various combinations of scaling parameters in Table 6. Figure 10 is a table of plots, where each plot shows the centerline velocity profiles for the experimental data, normalized by the various combinations of scaling parameters. The columns have different choices of origin, either that the effective origin is the core nozzle exit $\left(L_{1}\right)$ or is the upstream-most nozzle exit $\left(L_{t}\right)$. The rows use different choices of equivalent flow conditions, $M a$ and $T s R$. In all the plots the diameter chosen was that of the fully mixed, mass-average nozzle area of the flowing streams $\left(D e_{\mathrm{m}}\right)$. Visually, in all the plots there is a light grouping of the curves, but nothing nearly as good as using the fitted values for $\alpha$ and $\beta$ as was done in Figure 9. In the plots where the core nozzle was used as the origin (left column) the curves fall into two bands. The three cases whose curves are shifted to the right are 80010,86010 , and 86210. From Table 4 we see that these all have unheated cores. They are also flows with no significant ambient flow; however, there are three other $M_{\infty}=0$ flows in the larger group of lines, so this is not the distinguishing factor. Strangely, when the origin is chosen as the first upstream nozzle exit $\left(L_{t}\right)$ the two twostream cases, 86010 and 86210, remain outstanding from the rest of the group, as seen in the right-hand plots.

Figure 11 is the same as Figure 10 except that now the diameter has been chosen to be that of the equivalent total nozzle area, $D e_{\mathrm{t}}$. Again, the collapse is not as good as using the individually fitted $\alpha$ and $\beta \square \square \square \square \square \square$ the groups are tighter than when the mass-weighted diameter was used. The main standout is the lower left-hand plot, where the data collapses into two tight groups. In this plot the origin is the core nozzle exit L1, and the flow conditions are that of the fully mixed jet. The same three flows that didn't fall with the others are in the smaller group of lines, but they are joined by case 80030 , which is the hot core stream only. This flow is a heated jet case, voiding the possibly explanation for the difference. The only other feature these flows have in common is that they have little or no flow on the second and third streams. Unfortunately, there was not enough variation in the database to confirm that this is significant.

In summary, it seems that the best collapse comes from choosing the origin near the core nozzle and the diameter to be that of the total nozzle area. The choice of Ma and TsR was not too critical, although there may be a significant advantage to choosing the fully mixed conditions if the distinguishing feature that causes two distinct groupings can be determined. Otherwise, it is reasonable to choose the core velocity, or perhaps the core-ambient velocity, as the equivalent jet velocity for prediction of potential core and plume decay. 


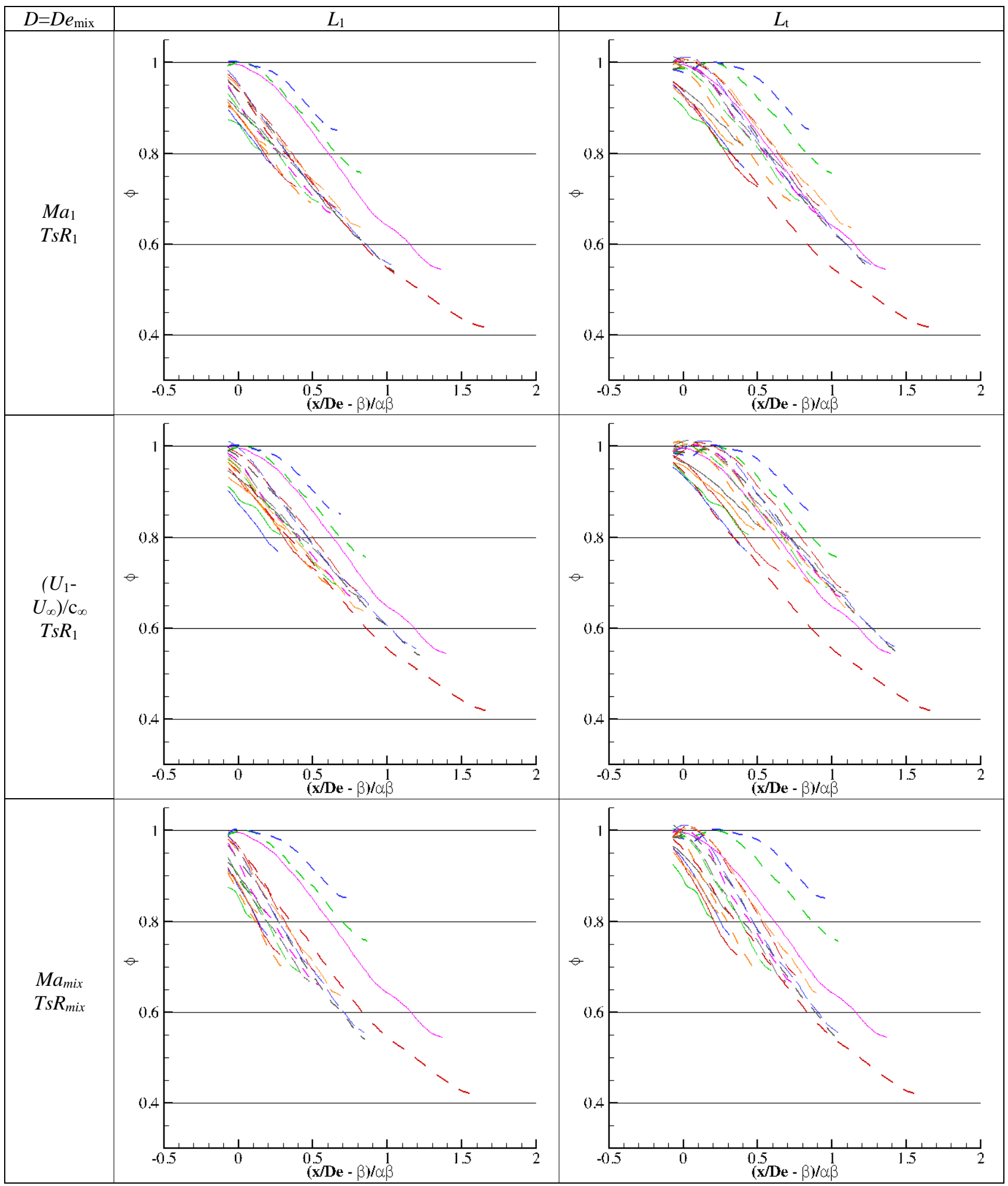

Figure 10 Collapse of mean velocities along centerline when normalized by coordinates given by origins $L_{1}$ and $L_{t}$ (left and right columns), diameter $D e_{\text {mix }}$, and coefficients $\alpha$ and $\beta$ computed using aggregate approximations for $M a$ and $T s R$ defined in Table 6 (rows). Line legend given in Figure 9.

12

American Institute of Aeronautics and Astronautics 


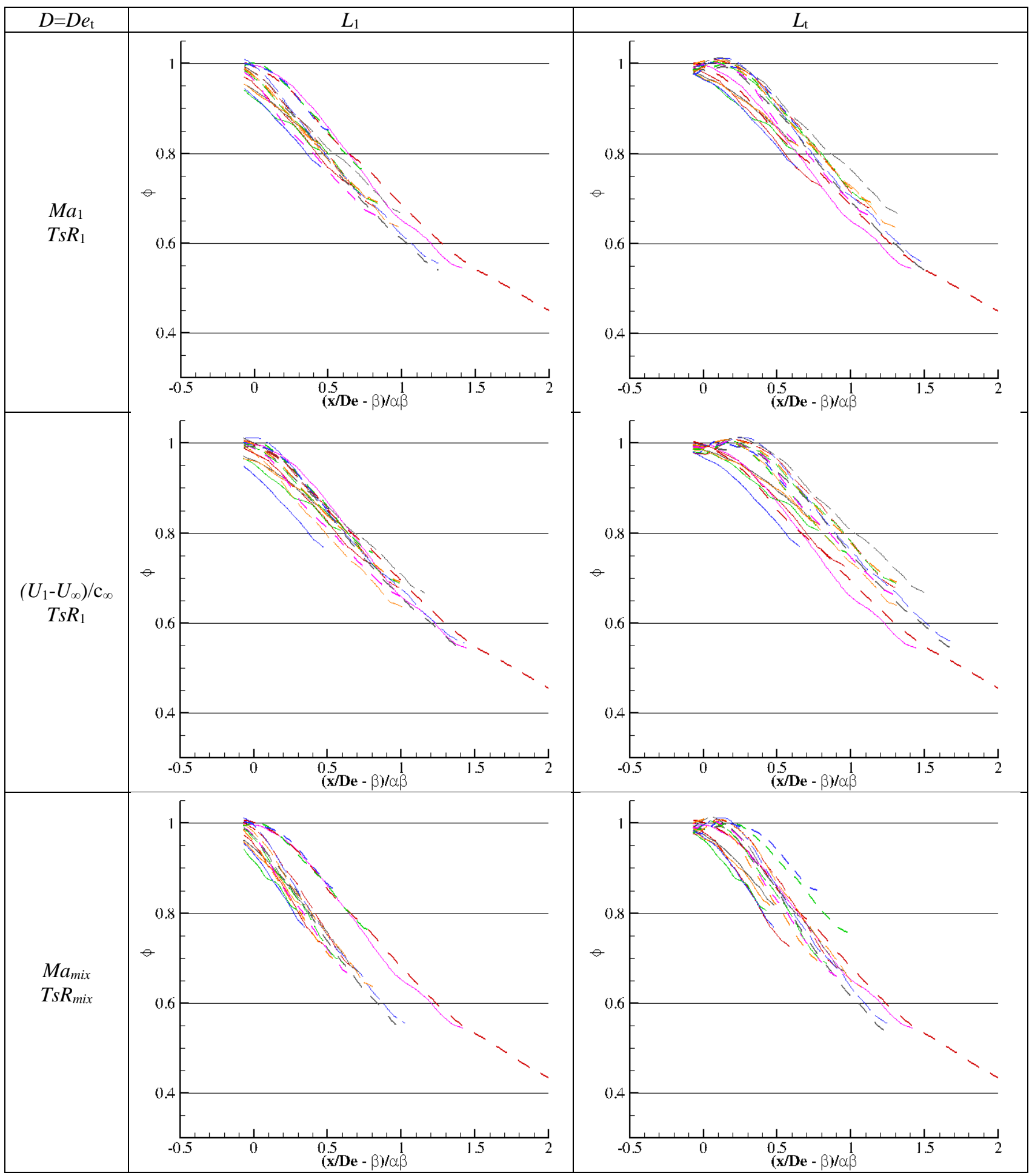

Figure 11 Collapse of mean velocities along centerline when normalized by coordinates given by origins $L_{1}$ and $L_{\mathrm{t}}$ (left and right columns), diameter $D e_{\mathrm{t}}$, and coefficients $\alpha$ and $\beta$ computed using aggregate approximations for $M a$ and $T s R$ defined in Table 6 (rows). Line legend given in Figure 9. 


\section{Comparisons of data to profiles predicted using the SSS model}

To directly visualize the ability of the SSS model to predict the centerline profiles of the multi-stream separate flow jets, a series of plots have been created to overlay the two. The SSS model coefficients and axial scaling have been chosen as follows:

- $U_{j}=$ maximum of the pseudo-centerline profiles (accounts for wake)

- $U_{\infty}=U_{\text {flight }}$

- $\quad D e=$ total area equivalent $\left(D e_{\mathrm{t}}\right)$

- $\quad x_{0}=$ core nozzle exit $\left(L_{1}\right)$

- $M a=M a_{\text {mix }}$

- $\quad T s R=T s R_{\operatorname{mix}}$

Using these values in equations [1] and [2] to predict the centerline velocity, comparisons were made with the extracted profiles, grouped by how well they agree with the SSS model.

In Figure 12 the model does an adequate job fitting the single-stream jets, both hot and cold, even though they have a plug. The decay region is not the best for the hot jet case, but the potential core is well captured. Recall that these were among the worst cases to collapse.

The model also does well for the low area ratio C3T3 nozzle cases (Figure 13), where the variations in third stream flow make a small impact. The area ratio of first to second stream is unity for the $\mathrm{C} 3$ nozzle, and the T3 third stream nozzle only adds another 0.6 to the cold stream area for a total area ratio of cold to hot flow of 1.6:1. The model captures the change in centerline profile through the difference in effective diameter, flow origin, and mass-weighted velocity.

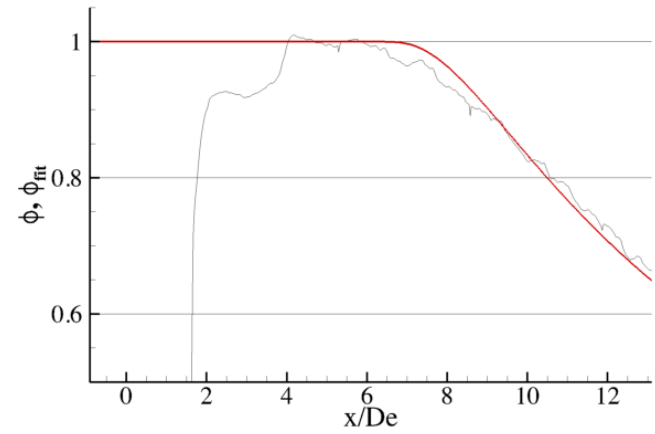

(a)

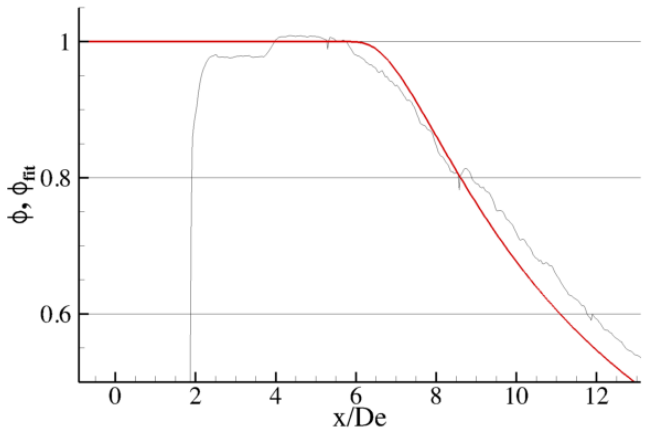

(b)

Figure 12 SSS model vs data for single-stream jet with plug. Centerline velocity profile for nozzle C3T1, core stream only, operating at (a) setpoint $80010\left(\mathrm{NPR}_{1}=1.8, \mathrm{NTR}=1\right.$, unheated $)$, and $(\mathrm{b})$ setpoint $80030(\mathrm{NPR} 1=$ 1.8, $\mathrm{NTR}_{1}=3$ ). 


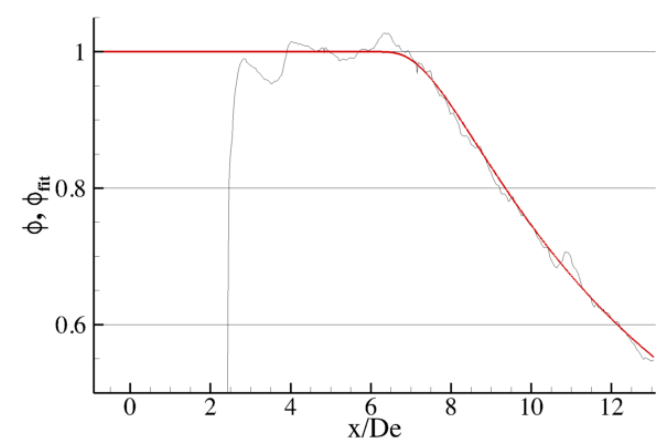

(a)

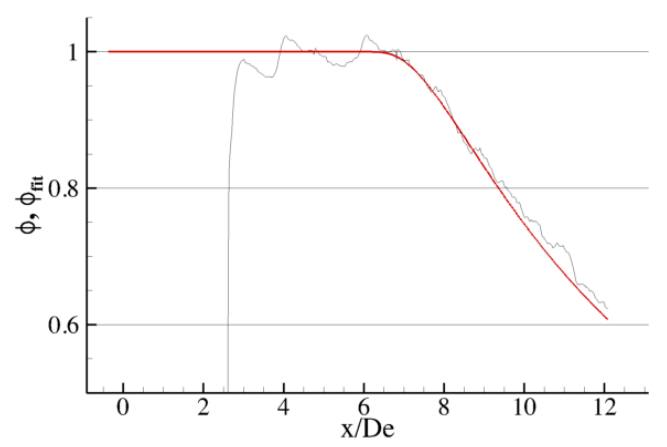

(b)

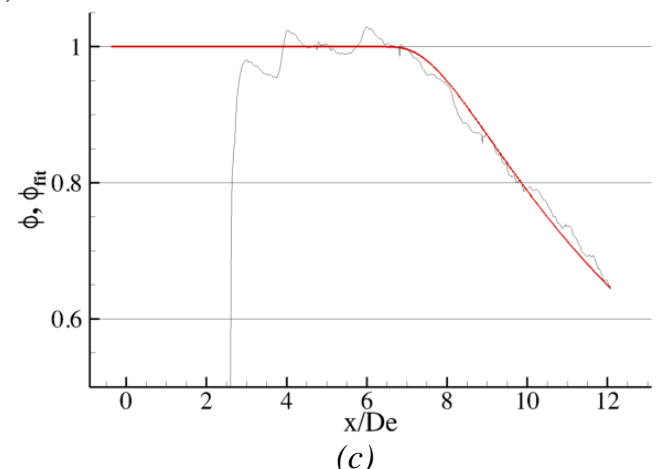

Figure 13 SSS model vs data for C3T3 nozzle (area ratio $A_{3} / A_{1}=0.6$ ), pressure matched on streams 1 and 2 $\left(\mathrm{NPR}_{1}=\mathrm{NPR}_{2}=1.8 ; \mathrm{NTR}_{1}=3\right)$, with various third streams. (a) setpoint $88033\left(\mathrm{NPR}_{3}=1.064\right.$ to match flight stream), (b) setpoint $88433\left(\mathrm{NPR}_{3}=1.4\right)$, (c) setpoint $88133\left(\mathrm{NPR}_{3}=2.1\right)$.

The C3T1 nozzle has a larger third stream area than C3T3, but only brings the cold to hot area ratio up to 2:1. The simple single stream model fits the data from this nozzle fairly well, but is beginning to overpredict the length of the potential core, especially as the third stream flow increases, as shown in Figure 14.

The success of the model is not universal, however, as shown in Figure 15. Here, the SSS model is compared for flows that are almost uniform in velocity profile, having cold flow on both core and bypass stream and a velocity ratio $U_{2} / U_{1}$ of 0.9 . Adding the low third stream flow did not make much difference in either the model or the data. Given the success of the model for the single-stream flow in Figure 12 this is a bit of a puzzle. 


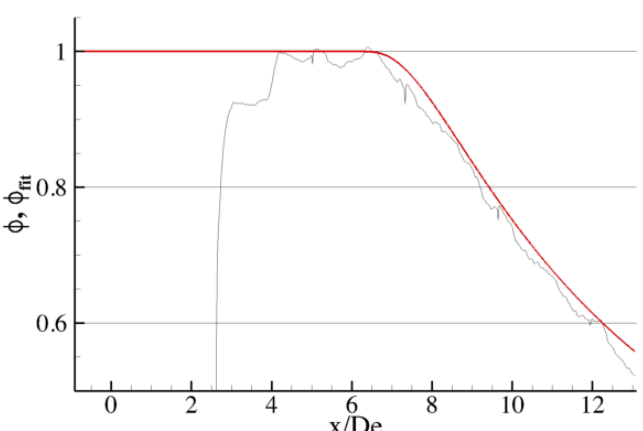

(a)

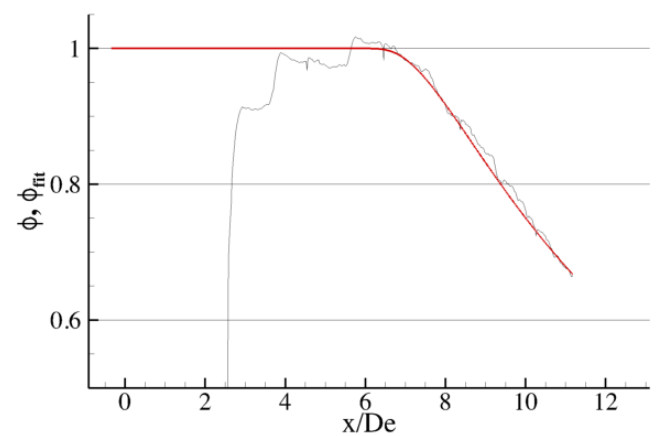

(c)

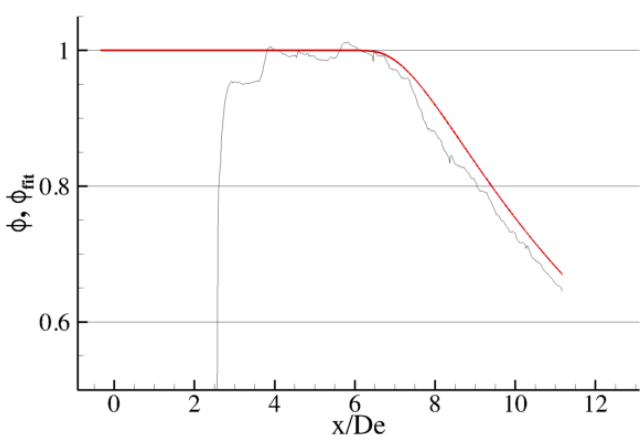

(b)

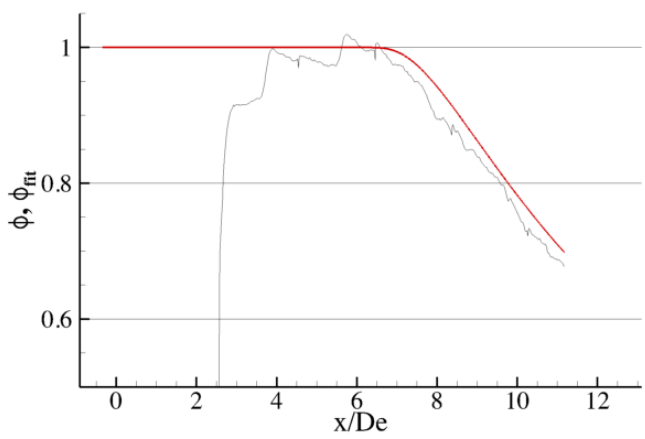

(d)

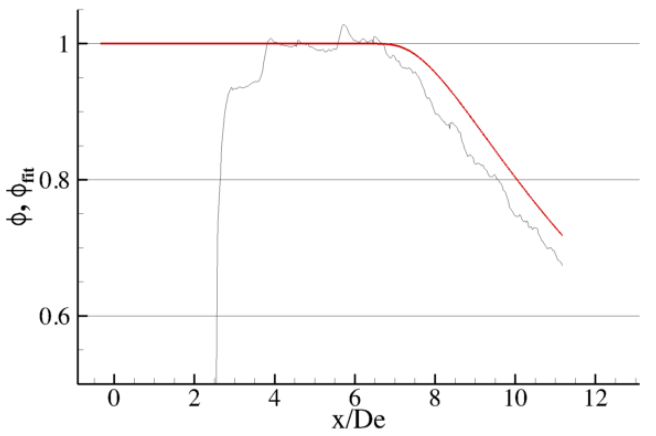

(e)

Figure 14 SSS model vs data for C3T1 nozzle (area ratios $A_{2} / A_{1}=1, A_{3} / A_{1}=1$ ), pressure matched on streams 1 and $2\left(\mathrm{NPR}_{1}=\mathbf{N P R}=1.8 ; \mathrm{NTR}_{1}=3\right)$, with various third streams and flight conditions. (a) setpoint $88033(\mathrm{NPR} 3$ $=1.064$ to match $M_{\infty}=0.3$ flight stream $)$, (b) setpoint $88430\left(\mathrm{NPR}_{3}=1.4, \mathrm{M}_{\infty}=0\right.$, no flight stream $)$, (c) setpoint $88433\left(\mathrm{NPR}_{3}=1.4, \mathrm{M}_{\infty}=0.3\right)$, (d) setpoint $88833\left(\mathrm{NPR}_{3}=1.8, \mathrm{M}_{\infty}=0.3\right)$, (c) $\operatorname{setpoint~} 88133\left(\mathrm{NPR}_{3}=2.1, \mathrm{M}_{\infty}=\right.$ 0.3).

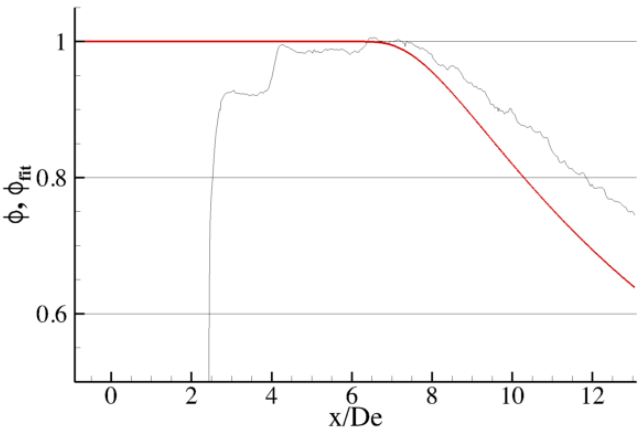

(a)

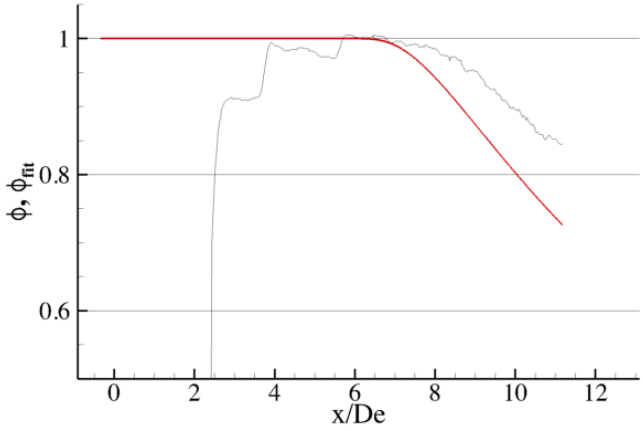

(b)

Figure 15 SSS model vs data for C3T1 nozzle with cold core $\left(N_{P R}=1.8\right)$, lower second stream $\left(N P R_{2}=1.6\right)$, $M_{\infty}=0$. (a) setpoint 86010 (no third stream flow), (b) setpoint $86210\left(\mathrm{NPR}_{3}=1.2\right)$. 


\section{About TKE}

In the end, the problem posed was whether one could predict the acoustically important features of the jet plume, such as the distribution of TKE. The SSS model, whose coefficients are based on fully mixed conditions, predicts that changing area ratio will make a significant difference in the length of the potential core, which we assume will change the location of the peak TKE. In the plots below the TKE fields are shown for area ratios 1:1 and 2.5:1 at the same flow conditions. In Figure 16 the fields are plotted in absolute coordinates (millimeters); in Figure 17 the axial coordinate is rescaled by total nozzle area $D_{t}$. In the latter plots the difference between the TKE of the two area ratios is minimal, making it seem that total nozzle area is the best choice for normalizing the axial coordinate to predict $T K E$ location. Unfortunately, the field of view in the PIV datasets was not large enough in downstream direction to clearly determine the location of the 'peak' TKE (in many cases the peak TKE was at the downstream end of the measured domain) so this statistic could not be constructed.

Contour lines of constant radial derivative of mean axial velocity have been drawn on the plots of Figure 16 and Figure 17. These allow us to see that the peak TKE aligns radially with the shear layer between the first and second streams, not the outer shear layer. This is somewhat counterintuitive since the axial location of the peak TKE scales on the potential core length, which is best correlated with either the outermost nozzle diameter $D e_{2}$ or the fully mixed jet dimensions $D e_{\mathrm{t}}$, not the diameter of the core nozzle $D e_{1}$.
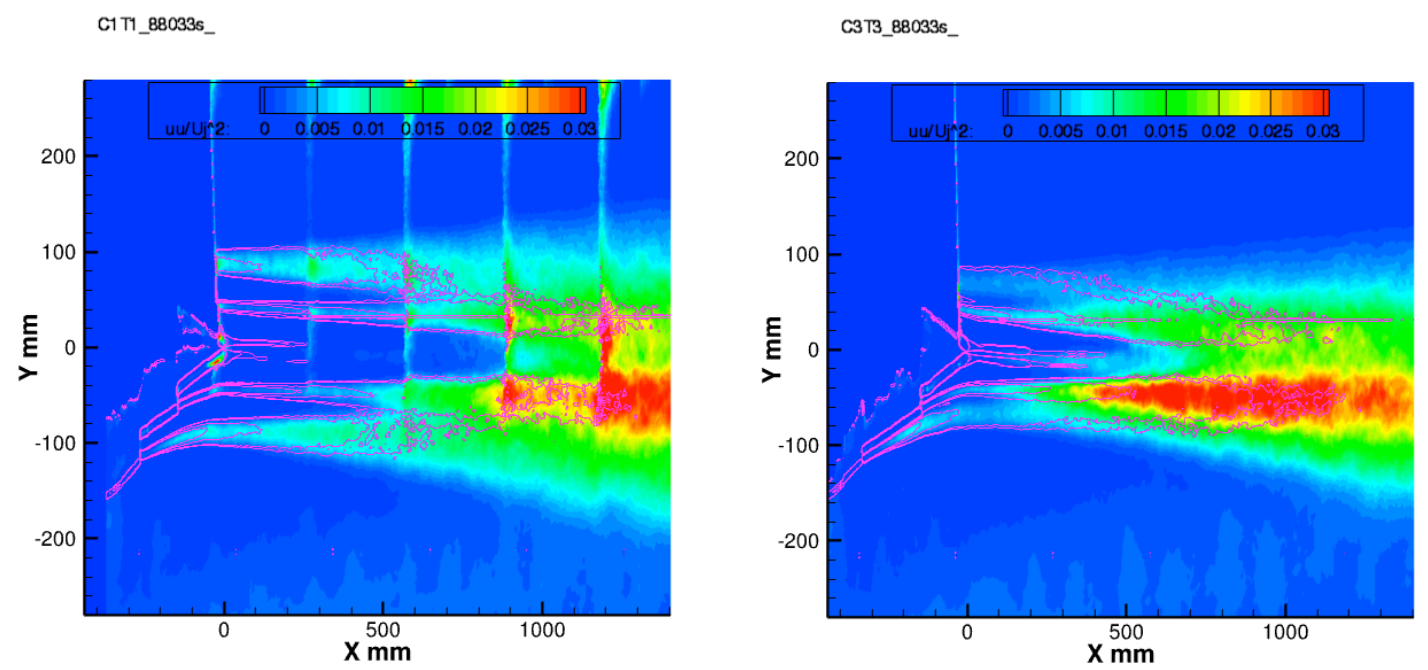

Figure 16 Comparison of flow field, mean gradient and TKE, on physical scale, for $\mathrm{AR}=1$ and 2.5 nozzles with setpoint 88033.
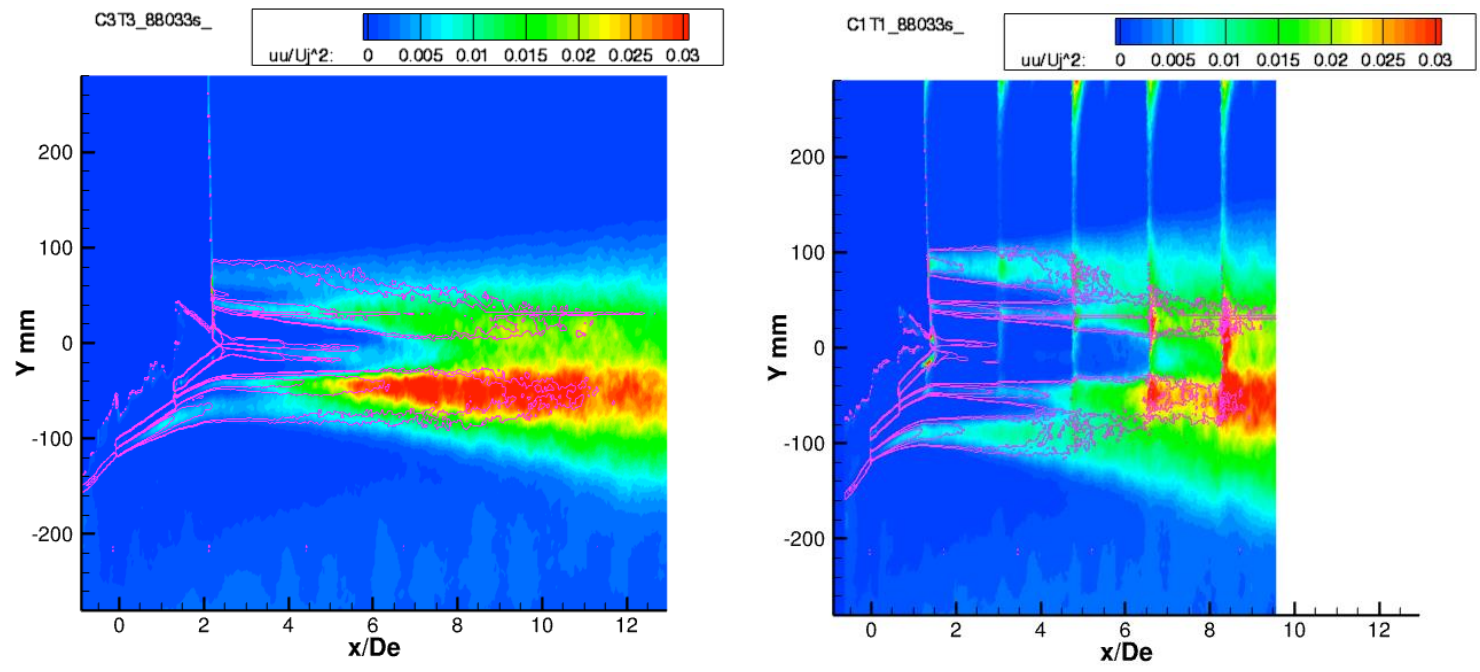

Figure 17 Comparison of flow field, mean gradient and $T K E$, on equivalent diameter basis, for $A R=1$ and 2.5 nozzles with setpoint 88033. $D e$ is diameter equivalent to total nozzle flow area, $D e t_{\text {t }}$ 
A careful measure of the radial location of peak TKE was carried out and, much like the extraction of the pseudocenterline data, the TKE was extracted along a max TKE radial line from the PIV data. A typical example of the line extracted from the data is illustrated in Figure 18. The radial location varied from $-30 \mathrm{~mm}$ to $-50 \mathrm{~mm}$. This corresponds most closely to the radius of an area equivalent to the core or inner nozzle $\left(D e_{1}=93 \mathrm{~mm}\right)$. The variations in radial locations were not found to correlate with any flow parameters. They are plotted versus $B P R$ in Figure 18 only for comparisons with previous measures of the jet, not because there is any particular trend with bypass ratio.
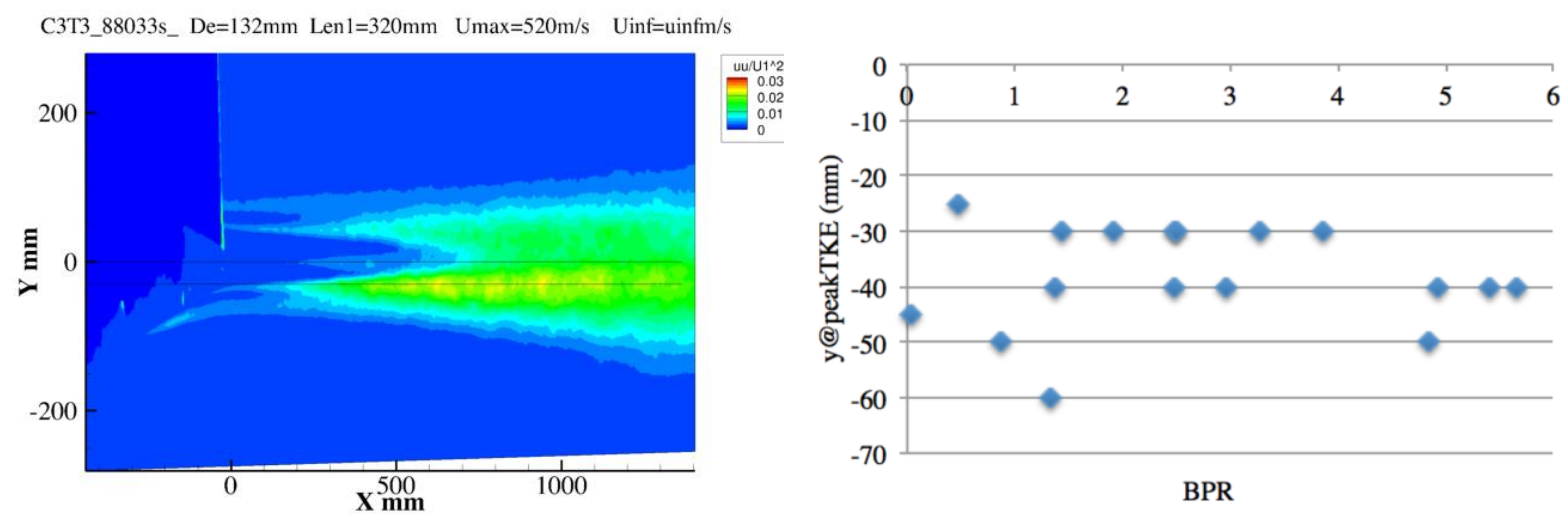

Figure 18 Radial line containing peak TKE. Left: sample plot showing location of peak TKE. Right: radial location of peak $T K E$.

Since the choice of equivalent diameter $D e_{t}$, based on total flow area, does the best job of collapsing the axial distribution of mean velocity and the location of the TKE, it would seem reasonable that a choice of fully mixed velocity would be the right velocity scale to collapse the TKE amplitude. On the other hand, it is the core nozzle diameter $D e_{1}$ that places the peak radially, so perhaps it is the velocity difference between the first two streams that scales the TKE amplitude. Neither assumption would be correct, however. Figure 19 compares the values of peak TKE as scaled by various choices of velocity or velocity difference. In the figure, normalizing the $T K E$ by fully mixed velocity (' $U_{\text {mix }}$ ') does not produce a consistent value for peak $T K E$. Even worse is when the normalizing velocity is taken to the difference between $U_{\text {mix }}$ and $\mathrm{U}_{\infty}$, the ambient velocity. The data collapses best when normalized on absolute value of the inner stream (' $U_{\max }$ ') than on any other combination of velocities, being nearly a constant value of 0.02 regardless of nozzle geometry or flow condition. Using a scaling velocity of the difference between the first stream and an outer stream does not improve the collapse. The TKE normalized by the velocity difference $\left(U_{1}-U_{\infty}\right)$ is plotted in Figure 19; however, to plot the TKE normalized by the velocity difference $\left(U_{1}-U_{2}\right)$ a larger scale had to be used. Figure 20 contains a plot of $T K E$ normalized by $\left(U_{1}-U_{2}\right)$, along with a plot of the $T K E$ normalized by $U_{1}$ ( $\left.=U_{\max }\right)$ to allow direct comparisons of the two best normalizations. The two normalizations are nearly identical in variance over the acquired database because most of the data had the same second stream velocity. However, the single-stream cases (lowest $B P R$ ) do not collapse using $U_{1}-U_{2}$. Hence, scaling on the velocity difference is not likely to produce a single non-dimensional value for $T K E$ when applied to jets with different second stream flow conditions. 


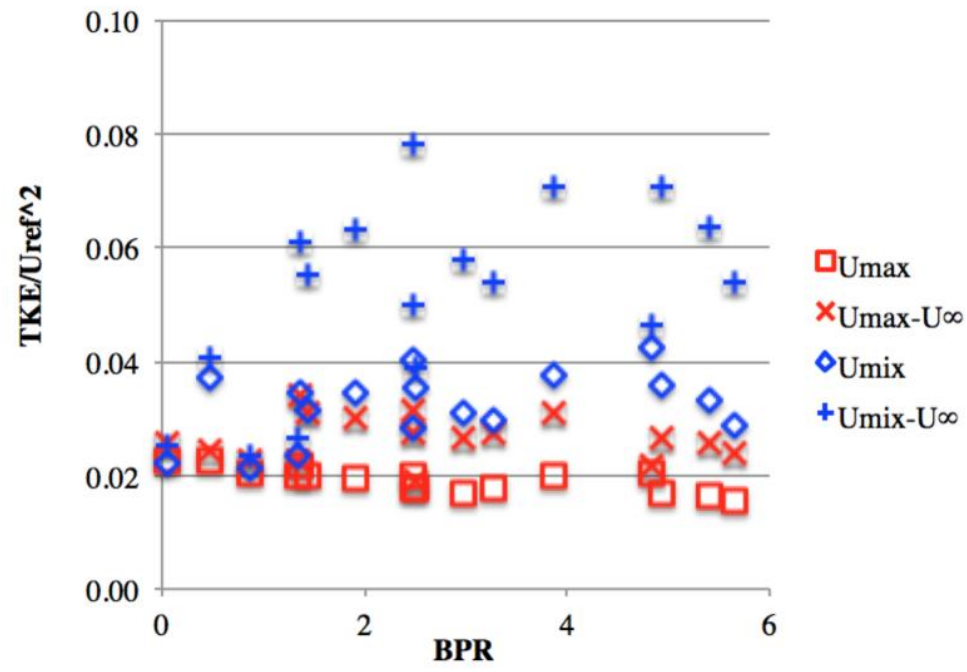

Figure 19 Peak TKE as normalized by various reference velocities, for all cases in the PIV test.
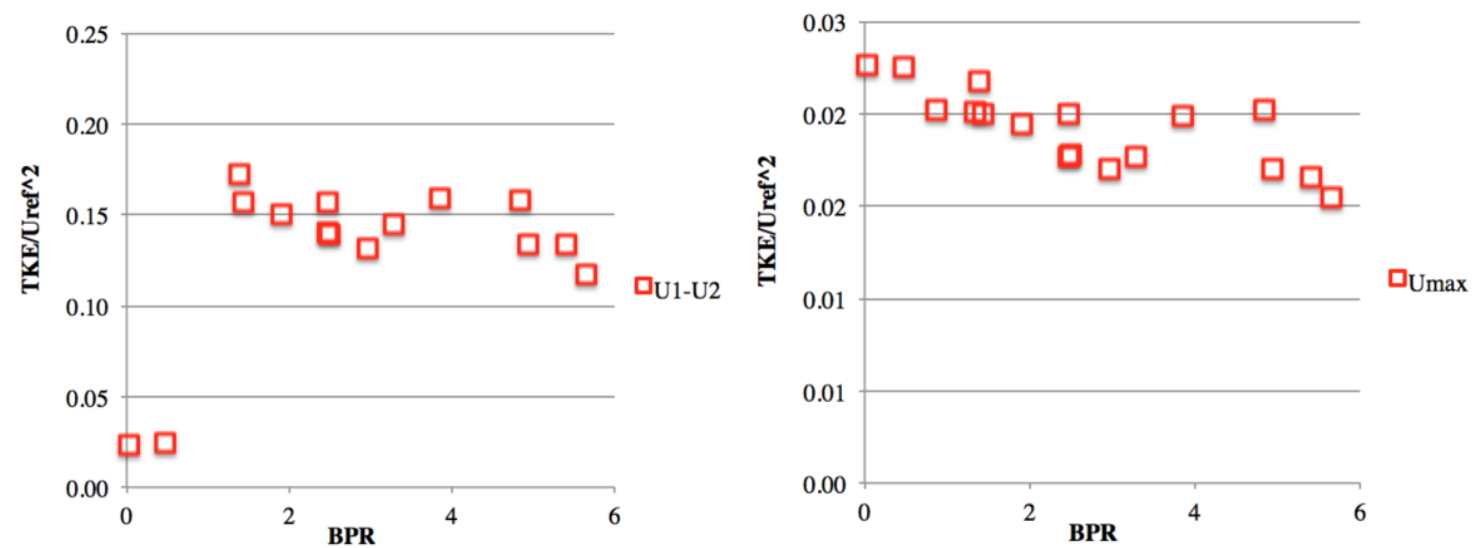

Figure 20 Peak $T K E$ as normalized by $\left(U_{1}-U_{2}\right)$ and by $U_{\max }\left(=U_{1}\right)$, for all cases in the PIV test.

Summarizing the attempts to collapse the TKE fields of the jets, it appears that the core stream exit velocity $U_{1}$ best scales the peak amplitude. The axial distribution is best collapsed using a jet diameter with equivalent area as the total nozzle exit area, with the origin taken as the exit plane of the first flowing nozzle. The radial location of the peak $T K E$ is roughly aligned with the first stream equivalent diameter, although there is some variation that does not readily scale with any flow parameters. Figure 21a plots the peak $T K E$, normalized by $U_{1}$, against axial coordinates normalized by the fitted parameters $\alpha_{\square}$ and $\beta_{\square}$ for all the multi-stream flows. Note that this is the same axial scaling that produced Figure 9 in which the mean velocities along the centerlines collapse extremely well. For comparison, the TKE is shown in Figure $21 \mathrm{~b}$ are plotted against the axial coordinate normalized by centerline decay parameters found by plugging fully mixed flow values into the SSS model $($ Eqs $[3,4])$. The corresponding plot of mean velocity decay is given in Figure 11. This plot reflects the accuracy in predicting TKE location and amplitude by using the SSS model with fully mixed values for the coefficients $\alpha$ and $\beta$ and normalizing the TKE amplitude by the core stream exit velocity. 


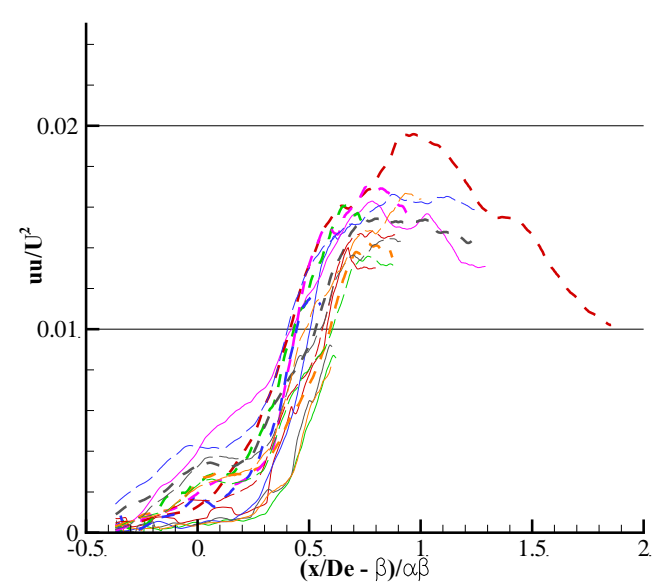

(a)

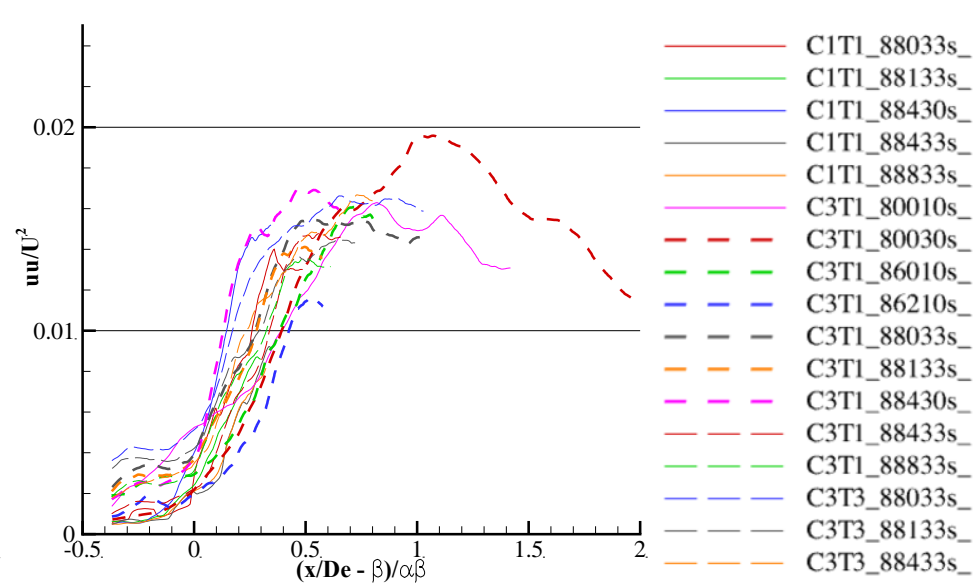

(b)

Figure 21 Collapse of $T K E$ along peak $T K E$ line when normalized by first stream velocity $U_{1}$ and plotted on axial coordinate normalized by (a) fitted coefficients $\alpha$ and $\beta$ using $L t, D e_{t}$ scaling of axial coordinate, and (b) SSS coefficients $\alpha_{\mathrm{M}}$ and $\beta_{\mathrm{M}}$ using $L t, D e_{\mathrm{t}}$ scaling of axial coordinate and fully mixed values of $M a_{\text {mixed }}$ and $T s R_{\text {mixed }}$ in Eqs $[3,4]$.

\section{Summary}

A relatively simple universal model has been demonstrated for the mean and turbulent velocities of single-flow jets, with two parameters determined by fitting of experimental data over a wide range of single-stream jets. The two parameters of this simple, single-stream (SSS) model were functions of jet exit diameter $D$, jet velocity (expressed as $M a=U_{j} / c_{\infty}$ ) and temperature (given by the ratio of jet static temperature to ambient temperature, $T_{j} / T_{\infty}$ ) and equate to a measure of the potential core length and the decay rate of the velocity profile. Data from multi-stream jets were similarly analyzed, and attempts at making simple approximations of their flow fields to that of the single-stream jets were demonstrated. The best choice of geometric and flow variables to make the SSS model predict the axial scaling of multi-stream jets seemed to be total nozzle area for the equivalent jet diameter and upstream-most nozzle exit as the origin. Of the various choices for $M a$ and $T_{j, s} / T_{\infty}$ in the SSS model for axial scaling, the fully mixed flow conditions were the best choices to equate the multi-stream jet with a single-stream jet. The amplitude scaling of the mean velocity profiles was best expressed as the velocity relative to the ambient velocity; however, the peak TKE amplitude scaled on the core velocity alone, not the fully mixed velocity or the initial velocity difference.

\section{Acknowledgements}

Obviously, this analysis would not have been possible without the data acquired by colleagues Drs Brenda Henderson and Mark Wernet, and their technicians. The Commercial Supersonics Technology Project of the Advanced Air Vehicles Program at NASA supported this work.

\section{References}

${ }^{1}$ Podboy, G.G., “Jet-Surface Interaction Test: Phased Array Noise Source Localization Results," GT2012-69801, ASME Turbo Expo 2012, (Copenhagen 2012).

${ }^{2}$ Brown, C.A., "An Empirical Jet-Surface Interaction Noise Model with Temperature and Nozzle Aspect Ratio Effects", AIAA 2015-0229, (Dallas 2015).

${ }^{3}$ Bridges, J, and Wernet, M.P. "Validating Large-Eddy Simulation for Jet Aeroacoustics,” J Propulsion Power 28, (2), pp 226-234, (2012).

${ }^{4}$ Witze, P.O. “Centerline Velocity Decay of Compressible Free Jets,” AIAA Journal, Vol. 12, No. 4, pp. 417-418 (1974).

${ }^{5}$ Lau, J.C. "Effects of Exit Mach Number and Temperature on Mean-Flow and Turbulence Characteristics in Round Jets," Journal of Fluid Mechanics, Vol. 105, pp. 193-218, (1981).

${ }^{6}$ Papamoschou, D., and Rostamimonjezi, S. "Modeling of Noise Reduction for Turbulent Jets with Induced Asymmetry." AIAA 2158 (2012). 
${ }^{7}$ Henderson, B.S., and Wernet, M.P., "Flow field measurements of three-stream jets," to be presented at AIAA SciTech2016.

${ }^{8}$ Bridges, J. and Wernet, M.P. "The NASA Subsonic Jet Particle Image Velocimetry (PIV) Dataset," NASA/TM2011-216807, (2011).

${ }^{9}$ Murakami, E, and Papamoschou, D., "Mean Flow Development in Dual-Stream Compressible Jets," AIAAJ 40 (6), (2002). 